\title{
Facial Recognition System combining Pulse Coupled Neural Network and Eigenfaces Principles
}

\section{Ramafiarisona Hajasoa Malalatiana}

Telecommunication- Automatic- Signal- Image- Research Laboratory/Doctoral School in Science and Technology of Enginneering and Innovation/ University of Antananarivo Antananarivo, Madagascar

\author{
Randriamitantsoa Paul Auguste \\ Telecommunication- Automatic- Signal- Image- Research \\ Laboratory/Doctoral School in Science and Technology of \\ Enginneering and Innovation/ University of Antananarivo \\ Antananarivo, Madagascar
}

\begin{abstract}
The objective of this work is to establish a facial recognition algorithm combining image processing by the pulse coupled neural network/PCNN and the Eigenfaces principle. The pulse coupled neural network is a neural network based on the visual system of mammals, the purpose of its use is the extraction of contours that characterize a face on a facial image. These contours have been coded into a single vector set or weight vector for each image by the Eigenfaces principle, the vectors thus obtained are used as the basis for representing the initial facial image in a facial recognition system.
\end{abstract}

Keywords-Facial Recognition; Image Processing; Neural Network; PCNN; Eigenfaces.

\section{INTRODUCTION}

There are several principles and algorithms dealing with facial recognition, most use defined points for recognition, here we will use the contours of the face on image. The contours will be transformed into a set of unique vectors by the principle of Eigenfaces to facilitate the processing of data and to reduce the database used without distorting the recognition.

\section{PULSE COUPLED NEURAL NETWORK}

PCNN/Pulse Coupled Neural Network is a biological model based on the visual cortex of mammals, proposed by Eckhorn, to solve the tasks related to image processing [1], [2].

The standard model of PCNN is defined by the following equations [3], [4], [5]:

$$
\begin{gathered}
F_{i j}(n)=S_{i j}+F_{i j}(n-1) \cdot e^{-\alpha_{F}} \\
+V_{F} \cdot(M * Y(n-1))_{i j} \\
L_{i j}(n)=L_{i j}(n-1) \cdot e^{-\alpha_{L}}+V_{L} \cdot(W * Y(n-1))_{i j} \\
U_{i j}(n)=F_{i j}(n) \cdot\left(1+\beta \cdot L_{i j}(n)\right) \\
Y_{i j}(n)= \begin{cases}1, & \text { si } U_{i j}(n)>\Theta_{i j}(n) \\
0, & \text { sinon }\end{cases} \\
\Theta_{i j}(n)=\Theta_{i j}(n-1) \cdot e^{-\alpha_{\Theta}}+V_{\Theta} \cdot Y_{i j}(n-1)
\end{gathered}
$$

$F_{i j}$ : Feeding input
$L_{i j}$ : Linking input

$U_{i j}:$ Internal activation

$\Theta_{i j}:$ Threshold

$n$ : The number of iterations

$S_{i j}$ : input image

$\mathrm{W}, \mathrm{M}$ : connection function

$Y$ : output

$V_{F}, V_{L}, V_{\Theta}$ : Magnitude scaling term

$\propto_{F}, \propto_{L}, \propto_{\Theta}$ : Decay term

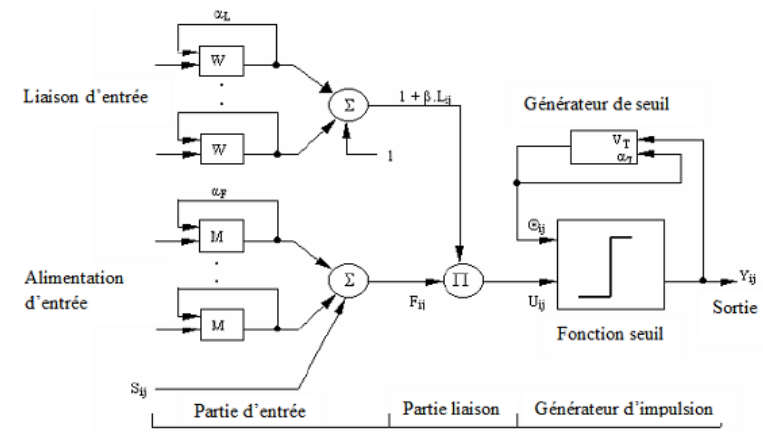

Fig. 1. PCNN's neuron model [1], [6], [7]

Our goal in using PCNN is to extract the contours of the face, mouth, and eyes.

\section{EIGENFACES}

By calculating the eigenvectors of the covariance matrix of the set of facial images, we have the Eigenvectors that define the variation between the facial images. Each pixel of the facial image contributes to each eigenvector, so we can display the eigenvectors as an image matrix called Eigenface [8], [9].

Each facial image of a set of images can be represented exactly as a linear combination of Eigenfaces. 
With the $\mathrm{M}^{\prime}$ best eigenfaces we have a sub-space of dimension M', the "face space", with which we can obtain every possible facial image by projection.

If we take a set of primary facial image including $\mathrm{M}$ images, $\Gamma_{1}, \Gamma_{2}, \Gamma_{3}, \ldots \ldots \ldots \ldots, \Gamma_{M}$.

The average between these set of images is defined by:

$$
\Psi=\frac{1}{M} \sum_{n=1}^{M} \Gamma_{n}
$$

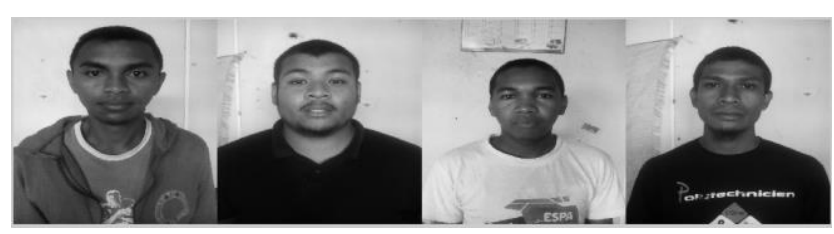

Fig. 2. Example of facial images

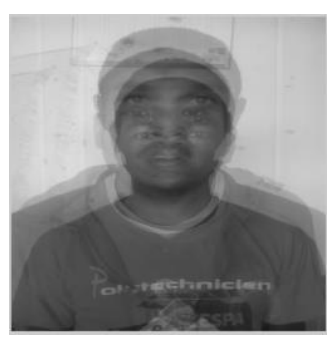

Fig. 3. Average

A facial image differs from this average by the vector:

$$
\Phi_{i}=\Gamma_{i}-\Psi
$$

This vector set will be subjected to a principal component analysis [9].

$$
\lambda_{k}=\frac{1}{M} \sum_{n=1}^{M}\left(u_{k}^{T} \Phi_{n}\right)^{2}
$$

$u_{k}$ and $\lambda_{k}$ are the eigenvectors and the eigenvalues of the covariance matrix $C$.

$$
C=\frac{1}{M} \sum_{i=1}^{M} \Phi_{i} \Phi_{i}^{T}=A A^{T}
$$

$A=\left[\Phi_{1}, \Phi_{2}, \ldots \Phi_{M}\right]$

Now let's take the eigenvectors $v_{i}$ of $A^{T} A$ :

$$
A^{T} A v_{i}=\mu_{i} v_{i}
$$

By multiplying each of the two sides by A:

$$
A A^{T} A v_{i}=\mu_{i} A v_{i}
$$

From which we can deduce that $v_{i}$ are eigenvectors of $C=A A^{T}$.

According to this analysis we have the matrix $L=A^{T} A$ of dimension MxM, where $L_{m n}=\Phi_{m}^{T} \Phi_{n}$, et we have the M eigenvectors $v_{l}$ of $\mathrm{L}$.These vectors define a linear combination of the $\mathrm{M}$ basic facial images, which form the eigenfaces $u_{l}$ [9].

$$
\mathrm{u}_{l}=\sum_{k=1}^{M} v_{l k} \Phi_{k}, \quad l=1, \ldots \ldots, M
$$

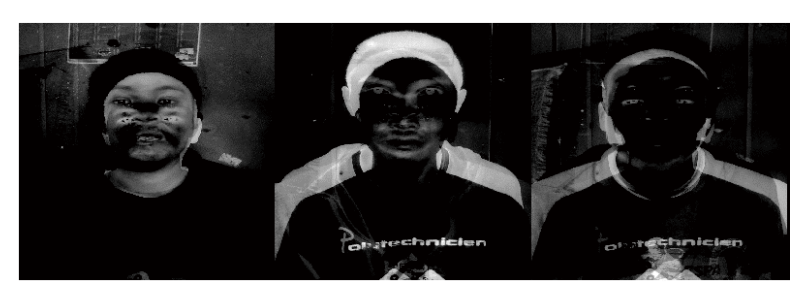

Fig. 4. Eigenfaces

Each normalized facial image $\Phi_{i}$ can be represented by the linear combination of the best eigenfaces:

$$
\begin{gathered}
\Phi_{i}=w_{j} \mathrm{u}_{j} \\
w_{j}=u_{j}^{T} \Phi_{i}
\end{gathered}
$$

$\mathrm{u}_{j}$ : eigenface and $\mathrm{w}_{j}$ : weight

To represent each facial image, we will keep only the best $\mathrm{K}$ eigenfaces, and we will calculate the vector $\Omega_{i}$.

$$
\Omega_{i}=\left[\begin{array}{c}
w_{1}^{i} \\
w_{2}^{i} \\
\cdots \\
w_{K}^{i}
\end{array}\right], \quad i=1,2, \ldots, M
$$

A new facial image $\Gamma$ is projected in the "face space" according to the formula:

$$
\omega_{k}=u_{k}^{T}(\Gamma-\Psi)
$$

$k=1, \ldots \ldots \ldots, M^{\prime}$

The weights resulting from the contribution of each eigenface in the representation of the image form the vector $\Omega$ :

$$
\Omega=\left[\begin{array}{c}
\omega_{1} \\
\omega_{2} \\
\ldots \\
\omega_{M \prime}^{\prime}
\end{array}\right]
$$

$\Omega$ can be used later to define the new image and be used in a facial recognition algorithm [9].

\section{SYSTEM COMBINING PCNN AND EIGENFACES}

To do this we will follow the following steps:

- Set up a facial image base

- Image processing with the PCNN.

- Calculate the vector $\Omega_{i}$ with Eigenface algorithm

These are the initialization steps of the system. A new image will go through the following steps for recognition: 
- Image processing with the PCNN.

- Projection of the contours image in the "face space" to obtain the vector $\Omega$.

- Calculates the Euclidean distance $\epsilon_{k}$.

$$
\epsilon_{k}=\left\|\Omega-\Omega_{k}\right\|
$$

- Conclusion if the individual of the new image is a known individual and facial image is contained in the initial base or not.

\section{RESULT}

A. Detection of the characteristic contours of the face

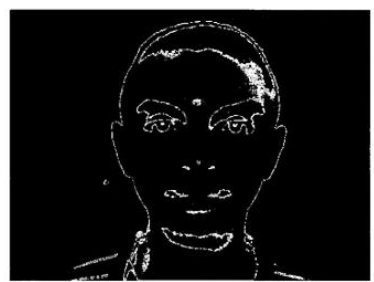

Fig. 5. Extracted contours

\section{B. Vector data base}

Each image representing the contours obtained is then used to calculate the Eigenfaces to obtain the "face sapce", and it is with these Eigenfaces that one can obtain by projection in the "face space" the unique vectors for each contour images.

$$
\begin{array}{rrrr}
1.0 \mathrm{e}+04 * & & & \\
0.6248 & 0.1914 & 0.3585 & 0.4632 \\
0.6179 & 0.2983 & 0.5595 & 0.7023 \\
0.6426 & 0.0899 & 0.4293 & 0.3383 \\
0.5220 & 0.2066 & 0.4039 & 0.3237 \\
0.1209 & 2.1570 & -2.4176 & -0.4596 \\
0.6108 & 0.3563 & 0.4533 & 0.7221 \\
0.7043 & 0.2393 & 1.3082 & -2.2925 \\
-5.7439 & -0.3937 & 0.0969 & -0.0086 \\
0.8059 & -0.2247 & 0.3487 & 0.5567 \\
1.0947 & -2.9202 & -1.5407 & -0.3455
\end{array}
$$

Fig. 6. Vector $\Omega_{k}$ matrix of 10 images

Our database for the biometric system is composed of this Vector $\Omega_{k}$ matrix and Eigenfaces.

\section{Evaluation}

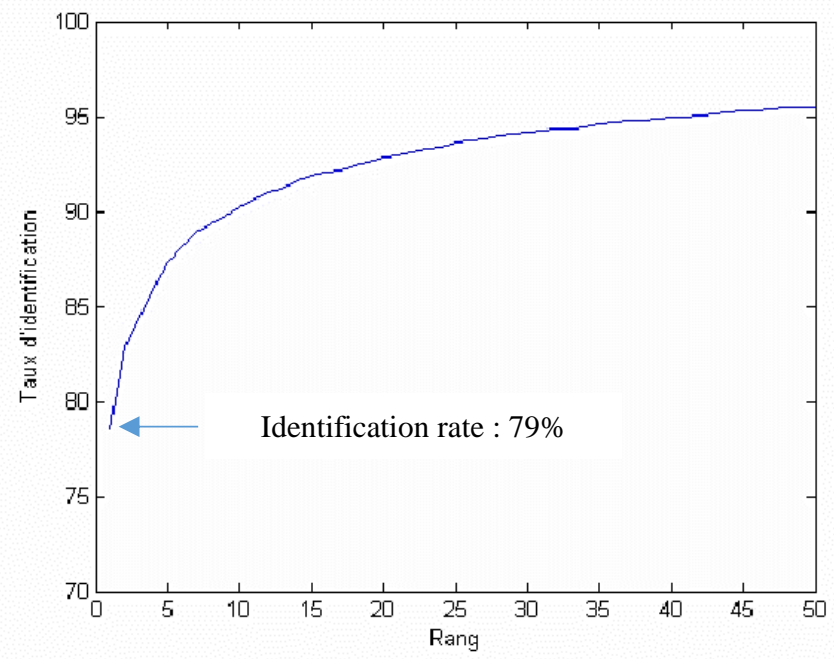

Fig. 7. Cumulative scoring curve of the realized system

This curve represents for each rank $n$ of abscissas the probability that the desired response is among those $n$ closest responses returned by the system.

\section{CONCLUSION}

PCNN is made for image processing in our case we exploited this network for the segmentation and detection of contours in facial images. These outlines being the information that interested us to represent the characteristics of the faces. After obtaining the contours of the facial images we have coded in a single vector each outlines image by the principle of Eigenfaces, on the one hand to create a database for a facial recognition system and on the other hand to facilitate the classification and identification of a facial image in a facial recognition system.

\section{REFERENCES}

[1] M. Rafidison, "Modélisation du couplage du traitement d'images numériques avec les réseaux de neurones", Thèse de Doctorat de l'ESPA, Spécialité : Images, 2014.

[2] C. Chatelain, "Réseaux de Neurones", Master IGIS Rouen, 09 Février 2012.

[3] M. Parizeau, “ Réseaux de Neurones ”, Université Laval, 2006.

[4] Z. Wang, Y. Ma, F. Cheng, L. Yang, "Review of pulse-coupled neural networks", School of Information Science and Engineering, Lanzhou University, Lanzhou, Gansu Province 730000, People's Republic of China, 2010.

[5] F. Pacifici, F. Frate, J. Emery, "Pulse coupled neural networks for automatic change detection in very high resolution images", Earth Observation Laboratory, DISP, Tor Vergata University, Rome, Italy, May 2009.

[6] T. Hoang, N. Nguyen, X. Nguyen, T. Bui, “A Real-time Image Feature Extraction Using Pulse-Coupled Neural Network", IJETTCS, October 2012.

[7] D. Fabio, "Pulse Coupled Neural Network for Automatic Features Extraction from Cosmo-Skymed and Terrasar-X Imagery", Tor Vergata University, 2009.

[8] D.A. Di Pietro, “ Analyse Numérique Matricielle ”, Notes de cours, A.A., 2012-2013.

[9] M.A. Turk, A.P. Pentland, "Face Recognition Using Eigenfaces", Vision and Modeling Group, The Media Laboratory, Massachusetts Institute of Technology, 1991. 